\title{
Escala para Avaliação de Estressores Psicossociais no Contexto Laboral: Construção e Evidências de Validade
}

\author{
Evaluation of Psychosocial Stressors in the Labor Context Scale: \\ Development and Psychometric Evidence
}

\author{
Maria Cristina Ferreira ${ }^{*}, a$, Taciano L. Milfont ${ }^{b}$, Ana Paula Corrêa e Silva ${ }^{c}$, \\ Helenita Araújo Fernandes ${ }^{d}$, Stanley Pacheco Almeida ${ }^{e} \&$ Helenides Mendonça $^{f}$ \\ ${ }^{a}$ Universidade Salgado de Oliveira, Niterói, RJ, Brasil, \\ ${ }^{b}$ Victoria University of Wellington, Wellington, Nova Zelândia, \\ ${ }^{c}$ Faculdades Integradas Maria Thereza, Niterói, RJ, Brasil, ${ }^{d}$ Movimento RH, Rio de Janeiro, RJ, Brasil, \\ ${ }^{e}$ Universidade Estácio de Sá, Rio de Janeiro, RJ, Brasil \\ \& ${ }^{f}$ Pontificia Universidade Católica de Goiás, Goiânia, GO, Brasil
}

\begin{abstract}
Resumo
Este estudo teve como objetivo desenvolver e verificar as evidências iniciais de validade e a consistência interna de uma Escala de Avaliação de Estressores Psicossociais no Contexto Laboral. Participaram do primeiro estudo 630 trabalhadores $($ mulheres $=51,5 \%$, média de idade $=32,77 ; D P$ $=10,33$ ) e, do segundo, 305 trabalhadores (mulheres $=44,3 \%$; média de idade $=34,62 ; D P=12,36$ ). As análises fatoriais exploratórias indicaram uma estrutura fatorial latente de sete fatores, com índices de consistência interna aceitáveis. Tal estrutura foi replicada na análise fatorial confirmatória. As correlações dos fatores com os afetos negativos dirigidos ao trabalho e a satisfação no trabalho foram, em geral, nas direções esperadas. Concluiu-se que a escala apresentou evidências iniciais de validade e consistência interna, o que recomenda seu uso futuro para fins de diagnóstico e pesquisa. Palavras-chave: Estresse ocupacional, estressores psicossociais, estudos de validação.
\end{abstract}

\begin{abstract}
The present study reports the development and psychometric parameters of the Evaluation of Psychosocial Stressors in the Labour Context Scale. Exploratory factor analysis conducted in Study 1 with data from 630 employees (females $=51.5 \%$; mean age $=32.77 ; \mathrm{SD}=10.33$ ) suggested a seven-factor structure for the 35 items, with good internal consistency indicators for all subscales. The seven-factor structure was supported with confirmatory factor analysis in another sample of 305 employees $(44.3 \%$ females, mean age $=43.62$; $\mathrm{SD}=12.36)$ in Study 2 . The correlations between the seven factors and both negative affects towards work and job satisfaction were overall consistent with predictions. This study provides initial evidence of validity and internal consistency for the new scale. The study concludes by recommending further psychometric tests of the scale as well as its future use for diagnosis and research.

Keywords: Occupational stress, psychosocial stressors, validation studies.
\end{abstract}

As mudanças na organização do trabalho têm levado ao aumento do estresse ocupacional ou laboral (Cooper, 2006), expressões usadas para designar não somente o processo, mas também o conjunto de reações físicas e psíquicas provocadas pela vivência de condições adversas no ambiente organizacional (Quick, Cooper, Nelson, Quick, \& Gavin, 2003). De fato, a exposição continuada às condições deflagadoras do processo de estresse pode levar o indivíduo a apresentar problemas comportamentais, médicos e psicológicos (Quick et al., 2003).

Endereço para correspondência: Universidade Salgado de Oliveira, R. Marechal Deodoro, 211, Bloco C, Centro, Niterói, RJ, Brasil 24030-060. Fone: (021) 2138-4926; Fax: (021) 2138-4926. E-mail: mcris@centroin.com.br, milfont@gmail.com, acaps1@gmail.com, helenita_f@hotmail.com, professorstanley@stanleypacheco.com e helenides@gmail.com
Embora o estresse ocupacional possa ter influência marcante na vida de trabalhadores de diversas carreiras e organizações, o desenvolvimento e validação de medidas psicométricas para a avaliação de tal fenômeno, com o apoio de modelos teóricos sólidos, tem sido escasso no contexto brasileiro. Nesse sentido, o presente artigo objetivou desenvolver e obter evidências iniciais de validade de uma Escala de Avaliação de Estresssores Psicossociais no Contexto Laboral.

\section{Os Modelos de Estresse Ocupacional}

Diferentes modelos têm sido propostos na literatura para explicar o estresse ocupacional. Tais modelos variam quanto ao número e à natureza dos fatores associados à etiologia do estresse, mas todos eles incluem os aspectos 
Ferreira, M. C., Milfont, T. L., Corrêa e Silva, A. P., Fernandes, H. A., Almeida, S. P. \& Mendonça, H. (2015). Escala para Avaliação de Estressores Psicossociais no Contexto Laboral: Construção e Evidências de Validade.

psicossociais presentes no contexto imediato do ambiente de trabalho como fundamentais à deflagração do estresse laboral. Os estressores psicossociais relacionam-se a características não físicas do ambiente ocupacional (Ganster, 2008), que exercem impacto na saúde física e mental, por meio de processos psicológicos decorrentes da interação dinâmica do indivíduo com seu contexto de trabalho (Ganster \& Rosen, 2013).

Um dos modelos mais referenciados na literatura sobre o estresse ocupacional é o de Cooper, Dewe e O'Driscoll (2001). Os autores propõem seis principais categorias de estressores ambientais de natureza psicossocial: fatores associados à natureza do trabalho; papeis organizacionais; relacionamento interpessoal no trabalho; insegurança na carreira; conflito trabalho-família e características organizacionais.

Os fatores associados à natureza do trabalho dizem respeito a aspectos inerentes ao contexto das tarefas desempenhadas pelo trabalhador, dentre os quais merecem destaque a sobrecarga que elas representam para o indivíduo e o grau de controle que ele exerce sobre elas. A sobrecarga de tarefas associa-se à extensão em que o empregado convive com demandas que excedem sua capacidade de trabalho (Cooper et al., 2001). O grau de controle no contexto do trabalho refere-se à autonomia ou liberdade que o empregado tem sobre as suas próprias tarefas, no que diz respeito ao planejamento e à tomada de decisões sobre elas (Karasek \& Theorell, 1990).

Os papeis organizacionais se relacionam às expectativas e exigências sobre os comportamentos adequados a uma determinada função (Beehr \& Glazer, 2005), e podem ser subdivididos em ambiguidade de papeis, conflito de papeis e responsabilidade de papeis (Cooper et al., 2001). A ambiguidade de papeis surge quando as pessoas não obtêm informações suficientes sobre as expectativas e exigências relativas a suas funções. $O$ conflito de papeis vincula-se às incompatibilidades entre as expectativas inerentes às diferentes funções que os empregados devem desempenhar. A pressão da responsabilidade pode ser subdividida em responsabilidade por coisas e responsabilidade por pessoas.

O relacionamento interpessoal no trabalho ou suporte social refere-se às interações positivas que os empregados mantêm com seus colegas e superiores (Kossek, Pichler, Bodner, \& Hemmer, 2011), as quais se revertem em ajuda emocional e instrumental. Tal ajuda pode se constituir em grande fonte de apoio, sendo que a falta dessa ajuda costuma levar ao aumento da sensação de pressão que gera o estresse ocupacional.

As fontes de estresse inerentes à insegurança na carreira vinculam-se à instabilidade sobre a manutenção do posto de trabalho e à percepção de falta de oportunidades de promoção, que impedem o desenvolvimento da carreira do indivíduo na organização (Cooper et al., 2001). Elas dizem respeito, portanto, às dificuldades de desenvolvimento profissional motivadas sobretudo pela falta de segurança na carreira.
O conflito trabalho-família relaciona-se à incompatibilidade entre as exigências dos papeis intrínsecos ao trabalho e as exigências dos papeis a serem desempenhados na família, ou vice-versa, o que costuma gerar pressão para o indivíduo (Greenhaus \& Beutell, 1985). As condições psicossociais vinculadas às características organizacionais diferem dos estressores anteriores, em função de se encontrarem em um nível macro de análise (organização), enquanto as outras fontes de estresse pertencem ao nível micro de análise. Tais características relacionam-se ao clima da organização, às políticas por ela adotadas e à sua cultura (Cooper et al., 2001).

No presente trabalho, somente as cinco categorias psicossociais de nivel micro, com suas respectivas subdivisões, foram adotadas como embasamento teórico para a construção do instrumento de estressores psicossociais do contexto laboral. A sexta categoria - características organizacionais - não foi incluída, em função de estar relacionada ao contexto organizacional e não ao contexto imediato do trabalho, razão pela qual tem sido avaliada por instrumentos de mensuração específicos.

$\mathrm{O}$ modelo teórico inicial que orientou a construção do instrumento compôs-se, portanto, de oito dimensões, retiradas do modelo de Cooper et al. (2001), e nomeadas no sentido de representarem um estressor em potencial. Foram elas: sobrecarga de tarefas; falta de autonomia; conflito de papeis; ambiguidade de papeis; pressão da responsabilidade; falta de suporte social; insegurança na carreira; conflito trabalho-família. As associações entre essas dimensões e reações individuais de natureza física e psicológica tem se evidenciado em diversos estudos, conforme preconizado pelo modelo de Cooper et al. (2001).

Nesse sentido, o conflito e a ambiguidade de papeis tem apresentado relações positivas com a exaustão emocional (Mulki, Jaramillo, \& Locander, 2008), e negativas, com a satisfação no trabalho (Chang, Rosen, \& Levy, 2009). A sobrecarga de trabalho tem se mostrado um forte preditor positivo da exaustão emocional (Demerouti, Baker, Nachreiner, \& Schaufeli, 2001), além de se associar negativamente à satisfação no trabalho (Mulki et al., 2008).

Associações positivas tem sido verificadas entre a falta de autonomia e o burnout, bem como negativas, entre tal estressor e a satisfação no trabalho (Luchman \& González-Morales, 2013). No que tange à falta de suporte social, relações positivas desse estressor com o burnout (Viswesvaran, Sanchez, \& Fisher, 1999), e negativas, com a satisfação no trabalho (Viswesvaran et al., 1999), vêm sendo observadas.

A insegurança na carreira tem se mostrado negativamente associada à satisfação no trabalho e à saúde física e psicológica, e positivamente, às intenções de rotatividade (Cheng \& Chan, 2008). Os conflitos trabalho-família têm se constituído em preditores negativos da satisfação no trabalho (Anderson, Coffey, \& Byerly, 2002) e positivos, do estresse geral percebido no trabalho (Hammer, Saksvik, Nytrø, Torvatn, \& Bayazit, 2004). 


\section{As Medidas de Estresse Ocupacional}

Diferentes instrumentos de autorrelato vêm sendo desenvolvidos e utilizados na avaliação das condições psicossociais do ambiente de trabalho associadas à deflagração do estresse ocupacional. Em revisão recente dessa literatura, conduzida nas bases de dados PubMed, PsychINFO, SSCI, Emerald, Science Direct, Ingenta Connect, Inter Science e Job Stress Network (usando-se as palavras-chave: psychosocial factors, work, stress, job, stressors, occupational, workplace, environment, health, questionnaire, instrument, measure, observational, subjective, objective, assessment), Tabanelli et al. (2008) identificaram 26 questionários dessa natureza.

No Brasil, as pesquisas sobre condições do ambiente de trabalho eliciadoras do estresse laboral têm se utilizado de instrumentos de autorrelato adaptados a partir de instrumentos desenvolvidos em outras culturas, bem como de instrumentos originalmente desenvolvidos no país. Em se tratando dos instrumentos adaptados ao contexto cultural brasileiro, os mais adotados têm sido o Job Content Questionnaire (JCQ) e a Job Stress Scale (JSS), ambos baseados no modelo de demanda-controle-suporte social (Karasek \& Theorell, 1990).

A versão padrão do JCQ foi desenvolvida por Karasek (1985) e validada no Brasil por Araújo e Karasek (2008). Segundo os autores, os resultados apontaram para uma estrutura de oito fatores, que se mostrou bastante similar à versão original, além de que a maioria das escalas apresentou índices de consistência interna adequados. No entanto, a escala de insegurança no trabalho não foi incluída na análise fatorial e os índices de consistência interna de várias escalas ficaram entre 0,60 e 0,70 , com duas delas apresentando valores abaixo de 0,60 .

A JSS é uma versão reduzida do JCQ, adaptada para o português do Brasil por Alves, Chor, Faerstein, Lopes, e Werneck (2004). A escala é usada com frequência em investigações brasileiras de diagnóstico de condições de trabalho, embora inexistam, até o momento, estudos capazes de atestar sua validade, além de que um de seus coeficientes de consistência apresentou-se relativamente baixo.

Quanto aos instrumentos originalmente brasileiros de avaliação do estresse no trabalho, merecem destaque a Escala de Estresse no Trabalho (EET) e a Escala de Vulnerabilidade a Estressores no Trabalho (EVENT). A EET (Paschoal \& Tamayo, 2004) foi submetida a procedimentos psicométricos que atestaram sua validade. Contudo, conforme apontado pelos próprios autores, ela é uma escala muito geral, que pode se mostrar pouco eficaz na avaliação dos efeitos isolados de diferentes categorias de estressores sobre as variadas reações ao estresse laboral.

A EVENT (Sisto, Baptista, Noronha, \& Santos, 2007) avalia a sensibilidade dos indivíduos a elementos estressores de seu ambiente de trabalho. Diferentes estudos psicométricos confirmaram a validade de construto e a precisão da EVENT (Sisto et al., 2007). Ela foi, porém, desenvolvida sem apoio de um modelo teórico sólido, o que fez com que as categorias que nortearam a elaboração inicial dos itens não tenham recebido apoio empírico, por ocasião da derivação final dos fatores componentes da escala.

Em síntese, os instrumentos que vêm sendo adotados mais frequentemente no Brasil para a avaliação das condições psicossociais do ambiente de trabalho potencialmente capazes de provocar o estresse ocupacional têm apresentado inconsistências. Justifica-se, dessa forma, a elaboração de novos instrumentos brasileiros de avaliação do construto em tela, que não incorram nos problemas apontados e que possam contribuir para a avaliação mais completa e sistemática das diferentes condições psicossociais de estresse presentes no ambiente de trabalho, bem como de seus efeitos sobre as múltiplas reações provocadas por tal processo de estresse. Fundamentando-se nessas considerações, e adotando como embasamento teórico o modelo de Cooper et al. (2001), o presente trabalho teve como objetivo geral desenvolver e buscar evidencias iniciais de validade, bem como analisar a consistência interna, de uma Escala de Avaliação de Estresssores Psicossociais no Contexto Laboral, por meio de dois diferentes estudos.

\section{Estudo 1}

Foi objetivo do Estudo 1 construir a Escala de Avaliação de Estressores Psicossociais no Contexto Laboral e verificar sua dimensionalidade, assim como a consistência interna de seus diferentes fatores.

\section{Método}

\section{Participantes}

O processo de amostragem foi por conveniência, e o único critério de inclusão foi o fato de as pessoas trabalharem há pelo menos um ano em qualquer tipo de organização. A amostra foi composta por 630 trabalhadores brasileiros de ambos os sexos (51,5\% de sexo feminino), pertencentes a 12 organizações do estado do Rio de Janeiro, provenientes dos setores da indústria $(n=2)$, comércio ( $n$ $=4)$ e serviços $(n=6)$, com idades variando entre $18 \mathrm{e}$ 70 anos (Média $=32,77 ; D P=10,33)$. A maioria $(80,0 \%)$ desses trabalhadores possuía nível de escolaridade superior completo ou incompleto, com o restante apresentando ensino médio completo.

\section{Instrumento}

Tomando por base as oito dimensões do modelo teórico de Cooper et al. (2001) que nortearam a elaboração do instrumento, foi inicialmente construída uma versão composta por 95 itens (positivos e negativos), retirados de instrumentos disponíveis na literatura da área (Cooper, Sloan, \& Williams, 1988; Spector \& Jex, 1998) ou criados para esse fim, a serem respondidos em escalas de seis pontos, variando de "nunca me afeta (1)" a "sempre me afeta (6)", segundo a frequiência com que cada estressor representava algo que provocava algum tipo de mal estar 
Ferreira, M. C., Milfont, T. L., Corrêa e Silva, A. P., Fernandes, H. A., Almeida, S. P. \& Mendonça, H. (2015). Escala para Avaliação de Estressores Psicossociais no Contexto Laboral: Construção e Evidências de Validade.

no trabalho (Exemplo de item: Receber instruções contraditórias sobre o que fazer no trabalho).

Ela foi submetida à análise de cinco juízes (estudantes de Mestrado em Psicologia, com experiência teórica e prática na área de trabalho, estresse e saúde mental há pelo menos dois anos), que avaliaram a pertinência de cada item a uma das oito dimensões prévias que orientaram a construção do instrumento. Os itens que obtiveram um índice de concordância de pelo menos $80,0 \%$ foram mantidos no instrumento, o que implicou a eliminação de cinco itens.

$\mathrm{Na}$ análise semântica, os 90 itens restantes foram aplicados a um grupo de dez trabalhadores de perfil semelhante aos incluídos na amostra final do estudo. Eles foram solicitados a apontar as dificuldades encontradas no preenchimento do questionário, no que se refere à compreensão dos itens e das instruções, o que levou à modificação de seis itens. Tal versão modificada, composta por 90 itens, foi adotada na coleta de dados da pesquisa, juntamente com um questionário sociodemográfico, com perguntas relativas ao sexo, idade, escolaridade e tipo de organização do respondente.

\section{Procedimentos}

O projeto de pesquisa foi aprovado pelo Comitê de Ética em Pesquisa da instituição dos autores. Em seguida, o instrumento foi aplicado em sessão única de tempo livre, sob a forma individual ou coletiva, em empresas e universidades, em seguida à obtenção de autorização dos responsáveis institucionais e à concordância de seus membros em colaborar com a pesquisa, mediante o preenchimento do termo de consentimento informado. Aos que concordaram em participar, foi garantido o anonimato.

$\mathrm{Na}$ análise dos dados, o atendimento aos pressupostos da análise fatorial exploratória foi verificado por meio do cálculo do KMO e do teste de Bartlett. Na identificação do número de fatores a serem extraídos, adotou-se a análise dos componentes principais e a análise paralela. Análises

Tabela 1 fatoriais dos eixos principais detectaram os itens a serem retidos em cada fator do instrumento.

\section{Resultados}

A medida de adequação da amostra de Kaiser-MeyerOlkin (KMO) foi de 0,94 , indicando a adequação da amostra à análise fatorial. $\mathrm{O}$ teste de esfericidade de Bartlett foi significativo $\left(\chi^{2}=24.888,54 ; p<0,001\right)$, mostrando que as correlações entre os itens permitiam a realização da análise fatorial.

A análise dos componentes principais extraiu 18 fatores com valores próprios maiores que 1 , responsáveis por $57,8 \%$ da variância total do instrumento. $\mathrm{O}$ teste gráfico (scree plot) revelou, porém, um número máximo de sete fatores interpretáveis, com a análise paralela também indicando a extração de sete fatores.

Em seguida, foi realizada uma análise fatorial exploratória dos eixos principais, com solução antecipada de sete fatores, por meio do método de rotação oblíqua, em virtude da expectativa de que os fatores se apresentariam correlacionados. Tais fatores explicaram $37,8 \%$ da variância total do instrumento, porém muitos dos itens do instrumento não apresentaram cargas fatoriais acima de $0,30 \mathrm{em}$ nenhum desses fatores.

Esses itens foram excluídos do instrumento e uma nova análise dos eixos principais foi conduzida, com os 47 itens restantes, a qual foi responsável por $40,7 \%$ da variância do instrumento. Para tornar o instrumento mais parcimonioso, foi selecionado, em cada fator, um total de quatro a seis itens (a depender do número inicial de itens retidos em cada fator), adotando-se os seguintes critérios: congruência teórica com a dimensão de origem do modelo que fundamentou o estudo; cargas fatoriais mais elevadas; maximização do índice de consistência interna (mínimo de 0,70 ). A Tabela 1 apresenta as cargas fatoriais dos itens selecionados para compor a versão final do instrumento e seus índices de consistência interna.

Resultados da Análise Fatorial Exploratória da Escala de Avaliação de Estressores Psicossociais no Contexto Laboral

\begin{tabular}{lllllllll}
\hline Itens & F1 & F2 & F3 & F4 & F5 & F6 & F7 \\
\hline
\end{tabular}

75. Receber instruções contraditórias sobre o que fazer no trabalho 0,77

76. Não saber exatamente a margem de autonomia que tenho em meu 0,67 trabalho

81. Não saber exatamente as tarefas que são de minha responsabilidade $\quad 0,65$

84. Não saber exatamente o que esperam de mim em meu trabalho $\quad 0,52$

66. Ser solicitado (a) a fazer coisas que vão contra meus próprios $\quad 0,38$ princípios

13. Trabalhar duro para cumprir minhas tarefas

22. Ter que trabalhar de forma coordenada com meus colegas de trabalho 
56. Ter um ritmo acelerado de trabalho

F 1

F1

52. Realizar várias tarefas ao mesmo tempo

18. Não receber ajuda de meus colegas quando tenho algum problema pessoal

27. Não receber ajuda de meus colegas quando tenho algum problema no trabalho

36. Não ser ouvido por meus colegas quando tenho dificuldades no trabalho

82. Não ser ouvido por meus colegas quando tenho algum problema pessoal

06. Não receber ajuda de meus superiores quando tenho algum problema pessoal

32. Não ser ouvido por meus superiores quando tenho algum problema pessoal

62. Conviver com rumores sobre cortes de pessoal

40. Conviver com rumores sobre dificuldades financeiras da empresa

02. Conviver com rumores sobre a possibilidade de fusão da empresa

74. Trabalhar em um ambiente de instabilidade quanto à minha permanência no emprego

70. Não poder planejar minhas horas de trabalho

46. Não poder decidir sobre quando me ausentar temporariamente de meu local de trabalho

57. Não poder decidir sobre quando fazer pausas durante meu horário de trabalho

54. Não poder estabelecer meu próprio ritmo de trabalho

50. Não poder decidir sobre quando tirar férias

58. Ter dificuldades de conciliar as questões profissionais com as familiares

39. Não poder cuidar direito de minha família em função do trabalho

09. Não dispor de tempo para minha vida pessoal em função do trabalho

30. Levar trabalho para casa

69. Não me desligar do trabalho mesmo quando estou em casa

53. Saber que meus erros podem prejudicar a mim mesmo (a)

71. Saber que meus erros podem interferir negativamente na vida de outras pessoas

24. Saber que meus erros podem ocasionar grandes perdas de equipamentos ou produtos

64. Saber que meus erros podem afetar o trabalho de outros colegas
0,55

0,54

0,42

0,73

0,66

0,65

0,58

0,56

0,55

0,71

0,55

0,48

0,43 
Ferreira, M. C., Milfont, T. L., Corrêa e Silva, A. P., Fernandes, H. A., Almeida, S. P. \& Mendonça, H. (2015). Escala para Avaliação de Estressores Psicossociais no Contexto Laboral: Construção e Evidências de Validade.

O Fator 1 compôs-se de cinco itens associados à falta de clareza sobre as próprias funções e ao recebimento de demandas contraditórias sobre as funções desempenhadas pelo empregado, e foi denominado de conflito $e$ ambiguidade de papeis. No Fator 2, concentraram-se seis itens associados ao excesso de tarefas que o empregado é solicitado, por vezes, a desempenhar, e foi rotulado de sobrecarga de papeis. O Fator 3 foi denominado de falta de suporte social, por estar relacionado a dificuldades advindas da falta de suporte emocional recebido pelos colegas e superiores, no cotidiano do trabalho, e compôsse de seis itens.

Quatro itens relativos a ameaças de demissão e à insegurança daí decorrente compuseram o Fator 4, intitulado de insegurança na carreira. Falta de autonomia foi o rótulo assinalado aos cinco itens do Fator 5, por ele estar relacionado às dificuldades de o empregado planejar $\mathrm{e}$ tomar decisões acerca de suas próprias tarefas. O Fator 6 foi rotulado de conflito trabalho/família, por implicar uma relação de incompatibilidade entre as responsabilidades associadas à família e ao trabalho, tendo ficado com cinco itens.

O sétimo e último fator ficou com quatro itens referentes ao grau de responsabilidade por pessoas e equipamentos que o empregado possui no desempenho de suas funções, tendo sido intitulado de pressão do grau de responsabilidade. Os percentuais de variância explicados por esses fatores foram iguais a $24,6 \%$ (conflito e ambiguidade de papeis), 6,3\% (sobrecarga de papeis), 3,4\% (falta de suporte social), 2,0\% (insegurança na carreira), 1,7\% (falta de autonomia), $1,4 \%$ (conflito trabalho/família) e 1,3\% (pressão do grau de responsabilidade).

\section{Discussão}

Os resultados obtidos revelaram que a estrutura interna da escala reproduziu quase que totalmente o modelo teórico inicial. Assim é que a versão final do instrumento ficou composta por 35 itens, distribuídos em sete fatores ou dimensões (conflito e ambiguidade de papeis; sobrecarga de papeis; falta de suporte social; insegurança na carreira; falta de autonomia; conflito trabalho-família; pressão do grau de responsabilidade), enquanto estavam inicialmente previstas oito dimensões.

A diferença entre as oito dimensões teóricas iniciais e as sete dimensões empiricamente derivadas deveu-se ao fato de que duas das dimensões iniciais (conflito de papeis e ambiguidade de papeis) se aglutinaram em um único fator ou dimensão, com os demais fatores reproduzindo o modelo original. Tal junção encontra respaldo empírico em estudos sobre o estresse ocupacional que vêm abordando conjuntamente essas dimensões (Lee \& Ashfort, 1996), em função de que na prática eles frequentemente se encontram associados (Liu, Liu, Mills, \& Fan, 2013).

As dimensões ora derivadas apresentam equivalencia conceitual com as dimensões constantes de outros instrumentos internacionais desenvolvidos para a avaliação de estressores psicossociais (Tabanelli et al., 2008). Tais dimensões têm sido também frequentemente adotadas nos estudos destinados a investigar as relações entre fontes e reações ao estresse, conforme apontado na recente revisão de Ganster e Rosen (2013) sobre estresse e saúde no trabalho. Os índices de consistência interna dessas dimensões variaram de 0,72 (insegurança na carreira) a 0,82 (dificuldades interpessoais), estando todos, assim, com valores superiores a 0,70 , o que é considerado aceitável.

A medida ora desenvolvida apresenta ainda a particularidade de não utilizar itens que fazem referencia conjunta à fonte (estressor) e à resposta ao estresse (problemas físicos, psicológicos e comportamentais). Tal estratégia tem sido criticada (Ganster, 2008), por confundir dois fenômenos distintos (fonte e reação ao estresse) em uma mesma medida, o que impede a análise mais acurada dos reais efeitos dos estressores sobre a saúde física e mental do indivíduo.

\section{Estudo 2}

O Estudo 2 objetivou confirmar a dimensionalidade do instrumento e obter indicadores de sua validade. De acordo com o modelo teórico de Cooper et al. (2001), os estressores psicossociais exercem impacto direto em reações físicas, psicológicas e comportamentais ao estresse. No entanto, segundo Ganster e Rosen (2013), esses estressores costumam apresentar relações mais fortes com reações psicológicas ao trabalho (exaustão emocional, satisfação no trabalho, afetos dirigidos ao trabalho) do que com reações físicas (distúrbios psicossomáticos). Tomando por base essas considerações, duas variáveis de natureza psicológica, mas com significados opostos (satisfação no trabalho e afetos negativos dirigidos ao trabalho), foram escolhidas para a busca de indicadores de validade da escala baseados nas correlações com outros construtos. A satisfação no trabalho expressa as crenças e julgamentos positivos acerca do próprio trabalho (Weiss, 2002) e, consequentemente, seria esperado que ela se mostrasse negativamente associada às sete dimensões de estressores. Os afetos negativos dirigidos ao contexto laboral consistem em respostas emocionais negativas que os indivíduos dirigem a seu contexto de trabalho (Ferreira, Silva, Fernandes, \& Pacheco, 2008) e, assim, seria esperado que as sete dimensões de estressores se mostrassem positivamente associadas a tais afetos.

\section{Método}

\section{Participantes}

A amostra foi de conveniência e o único critério de inclusão foi o fato de as pessoas trabalharem há pelo menos um ano em qualquer tipo de organização. Participaram da pesquisa 305 trabalhadores brasileiros, membros de três diferentes organizações (uma pública e duas privadas) situadas no estado do Rio de Janeiro. Desse total, 55,7\% 
eram homens, e suas idades variaram de 17 a 72 anos (Média $=34,62 ; D P=12,36$ ). Grande parte desses trabalhadores ocupava cargos administrativos ou técnicos $(53,4)$ e possuía nível de escolaridade universitário incompleto ou completo $(63,9 \%)$, com os demais possuindo ensino médio completo.

\section{Instrumentos}

Escala de Avaliação de Estressores Psicossociais no Contexto Laboral. A versão final da escala desenvolvida no Estudo 1 foi um dos instrumentos adotados na coleta de dados. Neste estudo, seus fatores apresentaram os seguintes coeficientes de consistência interna: conflito e ambiguidade de papéis $(0,77)$; sobrecarga de papéis $(0,71)$; dificuldades interpessoais $(0,77)$; insegurança na carreira $(0,62)$; falta de autonomia $(0,71)$; conflito trabalho/família $(0,75)$; pressão do grau de responsabilidade $(0,77)$. Quanto maior o resultado obtido em cada dimensão, maior a percepção de que ele representa uma fonte de tensão ou estresse para $\mathrm{o}$ indivíduo.

Escala de Satisfação no Trabalho. De autoria de Silva e Ferreira (2009), a escala se compõe de cinco itens, a serem respondidos em escalas de seis pontos, variando de "discordo fortemente (1)" a "concordo fortemente (6)", segundo o grau em que as pessoas manifestam sentimentos de satisfação ou insatisfação com seu trabalho. Exemplo de item: Sinto-me satisfeito com meu trabalho atual. A escala foi validada em uma amostra de trabalhadores, mediante análises fatoriais exploratórias que atestaram sua unidimensionalidade. A consistência interna da escala, no presente estudo, calculada pelo Alfa de Cronbach, foi de 0,88 . Quanto maior o escore na escala, maior o grau de satisfação do indivíduo com seu trabalho.

Escala de Afetos Negativos no Trabalho. Os afetos negativos no trabalho foram avaliados pela Escala de Afetos Negativos no Trabalho, que consiste em uma das subescalas da Escala de Afetos no Trabalho (ESAFE), de Ferreira et al. (2008), a qual consta de 14 itens, a serem respondidos em escalas de seis pontos, variando de "nunca (1)" a "sempre (6)". Exemplo de item: Meu trabalho me deixa nervoso. A validade do instrumento foi aferida em uma amostra de trabalhadores de instituições públicas e privadas, por meio de análises fatoriais exploratórias, nas quais se observou a presença de dois fatores (afetos positivos e negativos), e mediante o cálculo de correlações que forneceram evidências sobre a validade convergente e divergente das duas escalas. No estudo atual, o índice de consistência interna da Escala de Afetos Negativos foi de 0,90 . Quanto maior o resultado na escala, maior a frequência de afetos negativos dirigidos ao contexto do trabalho.

Questionário Sociodemográfico. Para a coleta de informações sociodemográficas da amostra foi utilizado um questionário com perguntas relativas ao sexo, idade, escolaridade, tipo de organização e função nela desempenhada pelo respondente.

\section{Procedimento}

O projeto de pesquisa foi aprovado pelo Comitê de Ética em Pesquisa da instituição dos autores. O instrumento foi aplicado em sessão única de tempo livre, sob a forma individual ou coletiva, somente em organizações, em seguida à obtenção de autorização dos responsáveis institucionais e à concordância de seus membros em colaborar com a pesquisa, mediante o preenchimento do termo de consentimento informado. Aos que concordaram em participar, foi garantido o anonimato.

$\mathrm{Na}$ análise dos dados, análises fatoriais confirmatórias foram realizadas para se investigar se a estrutura latente da Escala de Estressores Psicossociais no Contexto Laboral derivada no Estudo 1 seria replicada no Estudo 2. Para se verificar as correlações da escala com outros construtos a ela relacionados, foram calculadas as correlações $(r$ de Pearson) dos escores totais de cada um de seus fatores com os escores totais da Escala de Afetos Negativos e da Escala de Satisfação no Trabalho.

\section{Resultados}

A matriz de covariância entre os 35 itens da Escala de Avaliação de Estressores Psicossociais no Contexto Laboral foi submetida à análise fatorial confirmatória, por meio do método de Máxima Verossimilhança (Maximum Likelihood - ML) no LISREL 8.7. Todas as saturações (cargas fatoriais) mostraram-se fortes no fator correspondente, tendo variado de 0,37 a 0,72 , além de terem sido, sem nenhuma exceção, estatisticamente diferentes de zero $(t>1,96, p<0,05)$, o que indica sua adequação ao fator. O modelo teórico com sete fatores apresentou índices adequados de ajuste aos dados: $\chi^{2}(539)=949,23$, com indice subjetivo de ajuste $\left(\chi^{2} / g l\right)$ de 1,$76 ; \mathrm{CFI}=0,95 ; \mathrm{RMSR}=$ 0,$05 ;$ SRMR $=0,06$.

A análise das correlações entre os escores das escalas do estudo demonstrou que, atendendo ao que era previsto, as subescalas de conflito e ambiguidade de papéis $(r=$ $0,24 ; p<0,01)$, sobrecarga de papéis $(r=0,37 ; p<0,01)$, falta de suporte social $(r=0,30 ; p<0,01)$, insegurança na carreira $(r=0,24 ; p<0,01)$, falta de autonomia $(r=0,38$; $p<0,01)$ e conflito trabalho/família $(r=0,35 ; p<0,01)$ correlacionaram-se positiva e significativamente com a Escala de Afetos Negativos no Trabalho. Também conforme esperado, as subescalas de conflito e ambiguidade de papéis $(r=-0,19 ; p<0,01)$, sobrecarga de papéis $(r=-0,27$; $p<0,01)$, falta de autonomia $(r=-0,22 ; p<0,01)$ e conflito trabalho/família $(r=-0,19 ; p<0,01)$ correlacionaram-se negativa e significativamente com a Escala de Satisfação no Trabalho.

\section{Discussão}

Os resultados evidenciaram que todos os índices de ajuste do modelo, obtidos na análise fatorial confirmatória, foram satisfatórios, possibilitando, assim, a confirmação 
Ferreira, M. C., Milfont, T. L., Corrêa e Silva, A. P., Fernandes, H. A., Almeida, S. P. \& Mendonça, H. (2015). Escala para Avaliação de Estressores Psicossociais no Contexto Laboral: Construção e Evidências de Validade.

integral da estrutura fatorial derivada no primeiro estudo. Quanto às correlações da escala com outros construtos, observou-se que todas as subescalas de estressores se correlacionaram positiva e significativamente com os afetos negativos no trabalho, à exceção da subescala de pressão do grau de responsabilidade. Também conforme previsto, foram observadas correlações negativas e significativas das subescalas de conflito e ambiguidade de papéis, sobrecarga de papéis, falta de autonomia e conflito trabalho/ família com a satisfação no trabalho, com as três escalas de estressores restantes não apresentando correlações significativas com tal variável.

As relações negativas do conflito e ambiguidade de papéis, da sobrecarga de papéis, da falta de autonomia e do conflito trabalho-família com a satisfação no trabalho, bem como as relações positivas desses mesmos estressores com os afetos negativos dirigidos ao trabalho, confirmam estudos anteriores (Chang et al., 2009) que já reuniram evidencias sobre tais relações. Outrossim, elas corroboram o modelo teórico de Cooper et al. (2001), segundo o qual os estressores psicossociais constituem-se em antecedentes de respostas individuais de natureza psicológica, que trazem consequências para a saúde física e mental do indivíduo e para a organização.

A insegurança na carreira apresentou correlações positivas e significativas com os afetos negativos dirigidos ao trabalho, atendendo ao que era esperado, mas não se correlacionou com a satisfação no trabalho, conforme previsto. Tais resultados contrariam a meta-análise de Cheng e Chan (2008), que concluiu pela existência de relações negativas entre tal estressor e a satisfação no trabalho.

Borg e Elizur (1992) fazem distinção entre a insegurança na carreira de natureza cognitiva (percepção da probabilidade de perda de emprego) e a de natureza afetiva (preocupações e medos sobre a perda do emprego), sendo que Stiglbauer, Selenko, Batinic, e Jodlbauer (2012) observaram que a insegurança cognitiva correlacionava-se com o bem-estar cognitivo, mas não com o bem-estar afetivo. Como os itens da dimensão insegurança na carreira na atual escala são de natureza afetiva (refletem o mal-estar com situações associadas à instabilidade no emprego), é possível que por essa razão tal dimensão tenha se correlacionado com os afetos negativos, que também são de natureza puramente afetiva (Ferreira et al., 2008), mas não com a satisfação no trabalho, em função de tal reação ser de natureza predominantemente cognitiva (Weiss, 2002).

Outro estressor psicossocial que se correlacionou com os afetos negativos, conforme previsto, mas, contrariando o esperado, não se correlacionou com a satisfação no trabalho, foi a falta de suporte social. Os efeitos do suporte social sobre as reações ao estresse não são consensuais (Beehr, Bowling, \& Bennett, 2010), o que tem levado alguns autores (Baruch-Feldman, Brondolo, Ben-Dayan, \& Schwartz, 2002) a defender que o tamanho e a natureza de tais efeitos dependem de outras variáveis, como a fonte do suporte (supervisor ou colega de trabalho), por exemplo. No presente trabalho, porém, adotou-se uma concepção geral de suporte que não diferencia o suporte do supervisor e o de colegas. É possível, assim, que medidas mais gerais de suporte impactem principalmente reações afetivas (como é o caso dos afetos dirigidos ao trabalho), enquanto medidas especificamente relacionadas ao suporte do supervisor impactem em maior grau reações cognitivas (como é o caso da satisfação no trabalho).

A pressão da responsabilidade não se correlacionou com os afetos negativos dirigidos ao trabalho e nem tampouco com a satisfação no trabalho. Os estressores laborais podem ser categorizados em demandas de obstáculos e demandas de desafio (Crawford, LePine, \& Rich, 2010). As primeiras (conflito e ambiguidade de papéis, insegurança na carreira) dificultam a realização das tarefas e o alcance de metas, enquanto as últimas (complexidade das tarefas, pressão da responsabilidade) oferecem oportunidades para o desenvolvimento e a realização pessoal.

As demandas de obstáculos tendem a provocar reações emocionais negativas, ao passo que as demandas desafiadoras tendem a provocar reações emocionais positivas (Crawford et al., 2010). Logo, a pressão da responsabilidade pode não ter apresentado correlações significativas com os afetos negativos dirigidos ao trabalho em função de ela se constituir em uma demanda desafiadora que deveria, assim, apresentar correlações significativas com reações emocionais positivas. Ademais, a satisfação no trabalho pode não ter apresentado uma correlação positiva com a pressão da responsabilidade devido ao fato de ela se caracterizar como uma reação de natureza essencialmente cognitiva (Weiss, 2002). De todo modo, estudos futuros necessitam ser conduzidos para a confirmação de tais asserções.

\section{Considerações Finais}

Os índices psicométricos obtidos na análise da estrutura latente e da consistência interna da Escala de Estressores Psicossociais do Contexto Laboral, aliados aos indicadores acerca de suas relações com outros construtos, atestam que ela demonstrou ser um instrumento com evidências iniciais satisfatórias de validade de construto e fidedignidade. No entanto, a investigação apresentou limitações. Nesse sentido, ela não adotou critérios comportamentais externos que permitissem a verificação da validade preditiva da escala. Estudos futuros deveriam, portanto, avaliar esse aspecto, mediante a correlação dos diferentes estressores com critérios como o desempenho no trabalho, por exemplo.

Por outro lado, as amostras utilizadas em ambos os estudos apresentaram um perfil semelhante, caracterizado por trabalhadores de um único estado brasileiro e predominantemente com escolaridade superior. Torna-se assim necessária a realização de novos estudos destinados a obter evidências adicionais de validade em outros contextos ocupacionais e geográficos. A realização desses estudos poderá contribuir para o aprofundamento da compreensão acerca dos efeitos deletérios que o estresse ocupacional provoca nos indivíduos e nas organizações das quais fazem parte. 
Não obstante estas limitações, a escala apresentou uma estrutura fatorial mais robusta e extensa que as demais escalas nacionais congêneres. Ela revelou, ainda, melhores índices de consistência interna que duas das escalas adaptadas a partir de instrumentos desenvolvidos em outro contexto cultural e usadas no Brasil para avaliar os estressores psicossociais. Estes resultados indicam que a Escala de Estressores Psicossociais no Contexto Laboral apresenta propriedades psicométricas iguais ou superiores às medidas existentes para uso no Brasil, o que recomenda seu uso futuro em situações de diagnóstico e pesquisa dos aspectos psicossociais do contexto imediato do ambiente de trabalho capazes de deflagrar o estresse laboral.

\section{Referências}

Alves, M. G. M., Chor, D., Faerstein, E., Lopes, C. S., \& Werneck, G. L. (2004). Versão resumida da "Job Stress Scale": Adaptação para o português. Revista de Saúde Pública, 38,164-171. doi:10.1590/S0034-89102004000200003

Anderson, S. E., Coffey, B. S., \& Byerly, R. T. (2002). Formal organizational initiatives and informal workplace practices: Links to work-family conflict and job-related outcomes. Journal of Management, 28, 787-810. doi:10.1177/ 014920630202800605

Araújo, T. M., \& Karasek, R. (2008). Validity and reliability of the job content questionnaire in formal and informal jobs in Brazil. Scandinavian Journal of Work, Environment \& Health Supplements, 6, 52-59.

Baruch-Feldman, C., Brondolo, E., Ben-Dayan, D., \& Schwartz, J. (2002). Sources of social support and burnout, job satisfaction, and productivity. Journal of Occupational Health Psychology, 7, 84-93. doi:10.1037/1076-8998.7.1.84

Beehr, T. A., Bowling, N. A., \& Bennett, M. M. (2010). Occupational stress and failures of social support: When helping hurts. Journal of Occupational Health Psychology, 15, 45-59. doi:10.1037/a0018234

Beehr, T. A., \& Glazer, S. (2005). Organizational role stress. In J. Barling, E. K. Kelloway, \& M. R. Frone (Eds.), Handbook of work stress (pp. 7-33). Thousand Oaks, CA: Sage. doi:10.4135/9781412975995.n2

Borg, I., \& Elizur, D. (1992). Job insecurity: Correlates, moderators and measurement. International Journal of Manpower, 13, 13-26. doi:10.1108/01437729210010210

Chang, C., Rosen, C. C., \& Levy, P. E. (2009). The relationship between perceptions of organizational politics and employee attitudes, strain, and behavior: A meta-analytic examination. Academy of Management Journal, 52, 779-801. doi:10.5465/ amj.2009.43670894

Cheng, H. G., \& Chan, K. D. (2008). Who suffers more from job insecurity? A meta-analytic review. Applied Psychology: An International Review, 57, 272-303. doi:10.1111/j.14640597.2007.00312.x

Cooper, C. L. (2006). The changing nature of work: The new psychological contract and associated stressors. In A. M. Rossi, P. L. Perrewé, \& S. L. Sauter (Eds.), Stress and quality of working life: Current perspectives in occupational health (pp. 2-7). Greenwich, CT: Information Age.

Cooper, C. L., Dewe, P. J., \& O’Driscoll, M. P. (2001). Organizational stress: A review and critique of theory, research, and applications. Thousand Oaks, CA: Sage. doi:10.1093/ bjsw/32.5.648
Cooper, C., Sloan, S., \& Williams, S. (1988). Occupational stress indicator management guide. London: Thorbay.

Crawford, E. R., LePine, J. A., \& Rich, B. L. (2010). Linking job demands and resources to employee engagement and burnout: A theoretical extension and meta-analytic test. Journal of Applied Psychology, 95, 834-848. doi:10.1037/a0019364

Demerouti, E., Baker, A. B., Nachreiner, F., \& Schaufeli, W. B. (2001). The job demands-resources model of burnout. Journal of Applied Psychology, 86, 499-532. doi:10.1037/00219010.86.3.499

Ferreira, M. C., Silva, A. P. C., Fernandes, H., \& Pacheco, S. (2008). Desenvolvimento e validação de uma escala de afetos no trabalho. Revista de Avaliação Psicológica, 7, 143-150.

Ganster, D. C. (2008). Measurement challenges for studying work-related stressors and strains. Human Resource Management Review, 18, 259-270. doi:10.1016/j.hrmr.2008.07.011

Ganster, D. C., \& Rosen, C. C. (2013). Work stress and employee health: A multidisciplinary review. Journal of Management, 39, 1085-1122. doi:10.1177/0149206313475815

Greenhaus, J. H., \& Beutell, N. J. (1985). Sources of conflict between work and family roles. Academy of Management Review, 10, 76-88. doi:10.5465/amr.1985.4277352

Hammer, T. H., Saksvik, P. Ø., Nytrø, K., Torvatn, H., \& Bayazit, M. (2004). Expanding the psychosocial work environment: Workplace norms and work-family conflict as correlates of stress and health. Journal of Occupational Health and Psychology, 9, 83-97. doi:10.1037/1076-8998.9.1.83

Karasek, R. A. (1985). Job Content Questionnaire and user's guide. Lowell, MA: University of Massachusetts.

Karasek, R. A., \& Theorell, T. (1990). Healthy work: Stress, productivity, and the reconstruction of working life. New York: Basic Books.

Kossek, E. E., Pichler, S., Bodner, T., \& Hemmer, L. B. (2011). Workplace social support and work-family conflict: A meta analysis clarifying the influence of general and work-family specific supervisor and organizational support. Personnel Psychology, 64, 289-313. doi:10.1111/j.1744-6570.2011.01211.x

Lee, R. T., \& Ashfort, B. E. (1996). A meta-analytic examination of the correlates of the three dimensions of job burnout. Journal of Applied Psychology, 81, 123-133. doi:10.1037/00219010.81.2.123

Liu, C., Liu, Y., Mills, M. J., \& Fan, J. (2013). Job stressors, job performance, job dedication, and the moderating effect of conscientiousness: A mixed-method approach. International Journal of Stress Management, 20, 336-363. doi:10.1037/ a0034841

Luchman, J. N., \& González-Morales, M. G. (2013). Demands, control, and support: A meta-analytic review of work characteristics interrelationships. Journal of Occupational Health Psychology, 18, 37-52. doi:10.1037/a0030541

Mulki, J. P., Jaramillo, F., \& Locander, W. B. (2008). Effect of ethical climate on turnover intention: Linking attitudinal and stress theory. Journal of Business Ethics, 78, 559-574. doi:10.1007/s10551-007-9368-6

Paschoal, T., \& Tamayo, A. (2004). Validação da escala de estresse no trabalho. Estudos de Psicologia (Natal), 9, 45-52. doi:10.1590/s1413-294x2004000100006

Quick, J. C., Cooper, C. L., Nelson, D. L., Quick, J. D., \& Gavin, J. H. (2003). Stress, health, and wellbeing at work. In J. Greenberg (Ed.), Organizational behavior: The state of the science ( $2^{\text {nd }}$ ed; pp. 53-89). Mahwah, NJ: Lawrence Erlbaum.

Silva, A. P. C., \& Ferreira, M. C. (2009). Escala de Satisfação Geral no Trabalho [Resumo]. In Instituto Brasileiro de Avaliação Psicológica (Ed.), Anais do IV Congresso Brasileiro 
Ferreira, M. C., Milfont, T. L., Corrêa e Silva, A. P., Fernandes, H. A., Almeida, S. P. \& Mendonça, H. (2015). Escala para Avaliação de Estressores Psicossociais no Contexto Laboral: Construção e Evidências de Validade.

de Avaliação Psicológica (p. 246). Campinas, SP: Instituto Brasileiro de Avaliação Psicológica.

Sisto, F. F., Baptista, M. N., Noronha, A. P. P., \& Santos, A. A. A. (2007). Escala de vulnerabilidade a estressores no trabalho: Manual. São Paulo, SP: Vetor.

Spector, P. E., \& Jex, S. M. (1998). Development of four selfreport measures of job stressors and strain: Interpersonal conflicts at work scale, organizational constraints scale, quantitative workload inventory, and physical symptoms inventory. Journal of Occupational Health Psychology, 3, 356-367. doi:10.1037/1076-8998.3.4.356

Stiglbauer, B., Selenko, E., Batinic, B., \& Jodlbauer, S. (2012). On the link between job insecurity and turnover intentions: Moderated mediation by work involvement and well-being. Journal of Occupational Health Psychology, 17, 354-364. doi:10.1037/a0028565

Tabanelli, M. C., Depolo, M., Cooke, R. M. T., Sarchielli, G., Bonfiglioli, R., Mattioli, S., \& Violante, F. S. (2008). Available instruments for measurement of psychosocial factors in the work environment. International Archives of Occupational Environmental Health, 82, 1-12. doi:10.1007/ s00420-008-0312-6

Viswesvaran, C., Sanchez, J. I., \& Fisher, J. (1999). The role of social support in the process of work stress: A meta-analysis. Journal of Vocational Behavior, 54, 314-334. doi:10.1006/ jvbe.1998.1661

Weiss, H. M. (2002). Desconstructing job satisfaction: Separating evaluations, beliefs and affective experiences. Human Resource Management Review, 12, 173-194. doi:10.1016/ s1053-4822(02)00045-1 